PROCEEDINGS OF THE

AMERICAN MATHEMATICAL SOCIETY

Volume 139, Number 10, October 2011, Pages 3645-3656

S 0002-9939(2011)10784-6

Article electronically published on February 24, 2011

\title{
FINITE-DIMENSIONAL LEFT IDEALS IN THE DUALS OF INTROVERTED SPACES
}

\author{
M. FILALI AND M. SANGANI MONFARED \\ (Communicated by Mei-Chi Shaw)
}

\begin{abstract}
We use representations of a Banach algebra $A$ to completely characterize all finite-dimensional left ideals in the dual of introverted subspaces of $A^{*}$ and in particular in the double dual $A^{* *}$. We give sufficient conditions under which such ideals always exist and are direct sums of one-dimensional left ideals.
\end{abstract}

\section{INTRODUCTION}

Given a Banach algebra $A$, the dual space $A^{*}$ can be viewed as a Banach $A$ bimodule with the canonical operations

$$
\langle\lambda \cdot a, b\rangle=\langle\lambda, a b\rangle, \quad\langle a \cdot \lambda, b\rangle=\langle\lambda, b a\rangle,
$$

where $\lambda \in A^{*}$ and $a, b \in A$. Let $X$ be a norm closed $A$-submodule of $A^{*}$. Then given $\Psi \in X^{*}, \lambda \in X$, we may define $\Psi \cdot \lambda \in A^{*}$ by $\langle\Psi \cdot \lambda, a\rangle=\langle\Psi, \lambda \cdot a\rangle$. If $\Psi \cdot \lambda \in X$ for all choices of $\Psi \in X^{*}$ and $\lambda \in X$, then $X$ is called a (left) introverted subspace of $A^{*}$. The dual of an introverted subspace can be turned into a Banach algebra if for all $\Phi, \Psi \in X^{*}$ we define $\Phi \square \Psi \in X^{*}$ by $\langle\Phi \square \Psi, \lambda\rangle=\langle\Phi, \Psi \cdot \lambda\rangle$. In particular, by taking $X=A^{*}$, we obtain the first (or left) Arens product on $A^{* *}$, defined by Arens [1. The space $X^{*}$ can be identified with the quotient algebra $A^{* *} / X^{\circ}$, where $X^{\circ}=\left\{\Phi \in A^{* *}:\left.\Phi\right|_{X}=0\right\}$. If $X$ is faithful (that is, $a=0$ whenever $\lambda(a)=0$ for all $\lambda \in X)$, then the natural map of $A$ into $X^{*}$ is an embedding, and we will regard $A$ as a subalgebra of $\left(X^{*}, \square\right)$. The space $X^{*}$ has a canonical $A$-bimodule structure defined by $\langle a \cdot \Phi, \lambda\rangle=\langle\Phi, \lambda \cdot a\rangle,\langle\Phi \cdot a, \lambda\rangle=\langle\Phi, a \cdot \lambda\rangle\left(\Phi \in X^{*}, \lambda \in X, a \in A\right)$. One can then verify that $a \cdot \Phi=a \square \Phi, \Phi \cdot a=\Phi \square a$ for each $a \in A$ and $\Phi \in X^{*}$. We assume $X^{*}$ is equipped with the $w^{*}$-topology $\sigma\left(X^{*}, X\right)$. In this topology, for each $\Phi \in X^{*}$, the map $\Psi \mapsto \Psi \square \Phi, X^{*} \longrightarrow X^{*}$, is $w^{*}$-continuous.

In addition to $A^{*}$, other examples of introverted subpaces of $A^{*}$ include the space of left uniformly continuous functionals on $A$ defined by $L U C(A)=\overline{\operatorname{lin}}\left(A^{*} \cdot A\right)$ (the closure is in norm topology) and the space of [weakly] almost periodic functionals on $A,[W A P(A)] A P(A)$, defined as the set of all $\lambda \in A^{*}$ such that the linear map $A \longrightarrow A^{*}, a \mapsto a \cdot \lambda$, is [weakly] compact. If $G$ is a locally compact group, then $\operatorname{LUC}\left(L^{1}(G)\right)$ coincides with $L U C(G)$, the space of left uniformly continuous

Received by the editors March 31, 2010 and, in revised form, September 2, 2010.

2010 Mathematics Subject Classification. Primary 46H10, 46H15, 46H25, 43A20, 43A60.

The second author was partially supported by NSERC. 
functions on $G$ (cf. Lau [30]). For more information and additional examples one may consult $[8,9,10,20,27$.

It should be noted that even if $A$ is commutative, $X^{*}$ may not be commutative in general. The most notable cases of $X^{*}$ not being commutative are $L^{\infty}(G)^{*}$ for $G$ infinite, $\operatorname{LUC}(G)^{*}$ for $G$ non-compact, and $V N(G)^{*}$ and $U C_{2}(G)^{*}$ for $G$ non-discrete (cf. for example [15, 18, 29, 31, 32, 33, 35; these spaces are defined below). Ideals in $X^{*}$ (or in $A^{* *}$ ) have been previously studied by several authors, see for example Lau [28] for $A(G)^{* *}$, Ghahramani and Lau [19] for $L^{1}(G, w)^{* *}$ and $A_{p}(G)^{* *}$, Baker-Filali [3, 4, Filali [13, Filali-Pym [16, and Filali-Salmi [17] for (among other things) $X^{*}, L^{1}(G)^{* *}$, and $L U C(G)^{*}$, where $X$ is an introverted subspace of $C(G)$, Derighetti et al. [10] for duals of introverted subspaces of $p$ pseudo measures $P M_{p}(G)$, and the most recent work by the present authors and Neufang [14] on $A(G)^{* *}$ and $U C_{2}(G)^{*}$.

Let $G$ be a locally compact group, $1<p<\infty$, and $\mathscr{L}\left(L^{p}(G)\right)$ be the space of continuous linear operators on $L^{p}(G)$. Let $\lambda_{p}: M(G) \longrightarrow \mathscr{L}\left(L^{p}(G)\right)$ and $\lambda_{p}(\mu)(g)=$ $\mu * g$, where $\mu * g(x)=\int_{G} g\left(y^{-1} x\right) d \mu(y)$, be the left regular representation of $M(G)$ on $L^{p}(G)$. The space $P M_{p}(G)$ is the $w^{*}$-closure of $\lambda_{p}(M(G))$ in $\mathscr{L}\left(L^{p}(G)\right)$. This space is the dual of the Herz-Figà-Talamanca algebra $A_{p}(G)$, consisting of all functions $u \in C_{0}(G)$, such that $u=\sum_{i=1}^{\infty} g_{i} * \check{f}_{i}$, where $f_{i} \in L^{p}(G), g_{i} \in L^{q}(G)$, $1 / p+1 / q=1$, and $\sum_{i=1}^{\infty}\left\|f_{i}\right\|_{p}\left\|g_{i}\right\|_{q}<\infty$ (Herz [21]). When $p=2, A_{2}(G)$ and $\mathrm{PM}_{2}(G)$ coincide, respectively, with the Fourier algebra $A(G)$ and the group von Neumann algebra $V N(G)$ studied by Eymard in [12. In the following, for simplicity of notation, we denote $U C\left(A_{p}(G)\right)$ by $U C_{p}(G)$; when $p=2$, this space is also denoted by $U C(\widehat{G})$ in the literature.

Recently in [14] the present authors and M. Neufang proved the following theorem regarding left and right ideals in $A(G)^{* *}$ and in $U C_{2}(G)^{*}$.

Theorem 1.1. Let $G$ be a compact non-metrizable topological group and let $b(G)$ be the least cardinality of an open base at the identity. Suppose $b(G)$ has uncountable cofinality. Then:

(i) Every non-trivial right ideal in $A(G)^{* *}\left[U C_{2}(G)^{*}\right]$ has dimension at least $2^{2^{b(G)}}$

(ii) There are at least $2^{2^{b(G)}}$ closed left ideals of dimension at least $2^{2^{b(G)}}$ in $A(G)^{* *}\left[U C_{2}(G)^{*}\right]$.

This result is proved in [14, Theorems 4.3, 4.4]. The theorem shows that in general for a Banach algebra $A$, there may be no non-trivial, finite-dimensional right ideals in $A^{* *}$. In Filali-Pym [16] and Filali-Salmi 17] the authors showed a similar phenomenon for $L^{1}(G)^{* *}$ and $L U C(G)^{*}$ for any non-compact locally compact group $G$. In this paper we will show that the situation is different with regard to finite-dimensional left ideals in $A^{* *}$, or more generally in $X^{*}$, if $X$ is a faithful introverted subspace of $A^{*}$. In fact, using representations of $A$ we obtain complete characterization of finite-dimensional left ideals in $X^{*}$ (Theorems 2.7 and 2.8). We use our characterization to show that for commutative character amenable Banach algebras, if $X$ is a faithful introverted subspace of $A^{*}$, then every finite-dimensional left ideal in $X^{*}$ is a direct sum of 1-dimensional left ideals generated by $\phi$-invariant elements, where $\phi: A \longrightarrow \mathbf{C}$ is a character (Theorem 2.11).

In [13] the first-named author proved that finite-dimensional left ideals exist in $L^{1}(G)$ and $M(G)$ if and only if $G$ is compact. In the last section of this paper 
we obtain a dual version of this result in the context of commutative, regular, semisimple Banach algebras (which include many of the algebras of interest in harmonic analysis such as $C^{b}(G), L U C(G), A_{p}(G)$, Fourier-Stieltjes algebra $B(G)$, ...). For a general study of ideals in regular, commutative semisimple Banach algebras from the point of view of spectral synthesis, we refer to Section 39 of Hewitt and Ross [22].

We denote the set of all non-zero characters (that is, non-zero multiplicative linear functionals) on $A$ by $\sigma(A)$. Given $\phi \in \sigma(A) \cup\{0\}$, let us denote by $\mathcal{M}_{\phi}^{A}$ the set of all Banach $A$-bimodules $E$ such that the right module action of $A$ on $E$ is defined by $x \cdot a=\phi(a) x(a \in A, x \in E)$. We call $A$ (left) $\phi$-amenable if every continuous derivation $d: A \longrightarrow E^{*}$ is an inner derivation, for all $E \in \mathcal{M}_{\phi}^{A}$ (Kaniuth-Lau-Pym 26]). If the above condition holds for all choices of $\phi \in \sigma(A) \cup\{0\}$, then $A$ is called (left) character amenable (Monfared [37). Examples of character amenable Banach algebras include $A_{p}(G)$ and $L^{1}(G)$ when $G$ is an amenable locally compact group. We also recall that if $\phi \in \sigma(A) \cup\{0\}$, then an element $\Phi \in A^{* *}$ is called (left) $\phi$-invariant if $\Phi \cdot a=\phi(a) \Phi(a \in A)$ (a similar definition applies if $\Phi \in X^{*}$ ). It is known that if $A$ is character amenable, then for every $\phi \in \sigma(A), A^{* *}$ has a $\phi$-invariant element $\Phi$ such that $\Phi(\phi) \neq 0$ (see [37]). For more information on character amenability we refer to [24, 26, 37.

\section{IdEALS IN DUALS OF INTROVERTED SPACES}

Throughout this section $A$ denotes a Banach algebra and $X$ a faithful, introverted subspace of $A^{*}$. Let $H$ be a Hilbert space and $\mathscr{L}(H)$ be the space of continuous linear operators on $H$. Unless otherwise stated, we assume $\mathscr{L}(H)$ is equipped with the weak operator topology. This topology coincides with the norm topology if $H$ is finite-dimensional.

Let $\pi: A \longrightarrow \mathscr{L}(H)$ be a continuous representation of $A$. For each $\xi, \eta \in H$, the function $\pi_{\xi, \eta} \in A^{*}$ defined by $\left\langle\pi_{\xi, \eta}, a\right\rangle=(\pi(a) \xi \mid \eta)$ is called the coordinate function of $\pi$ with respect to $\xi, \eta$.

Definition 2.1. A continuous representation $\pi: A \longrightarrow \mathscr{L}(H)$ is called subordinate to $X$ if $\pi_{\xi, \eta} \in X$ for all $\xi, \eta \in H$.

If $\pi$ is subordinate to $X$, then the map

$$
\tilde{\pi}: X^{*} \longrightarrow \mathscr{L}(H), \quad(\widetilde{\pi}(\Phi) \xi \mid \eta)=\left\langle\Phi, \pi_{\xi, \eta}\right\rangle,
$$

where $\Phi \in X^{*}$, and $\xi, \eta \in H$ is well defined and is a $w^{*}$-continuous representation of $X^{*}$ on $H$. Note that if $\pi: A \longrightarrow M_{n}(\mathbf{C})$ is finite-dimensional, $\left\{\mathbf{e}_{1}, \ldots, \mathbf{e}_{n}\right\}$ is the standard basis of $\mathbf{C}^{n}$, and $\pi_{i j}=\pi_{\mathbf{e}_{j}, \mathbf{e}_{i}}$, then

$$
\widetilde{\pi}(\Phi)=\left(\begin{array}{ccc}
\Phi\left(\pi_{11}\right) & \cdots & \Phi\left(\pi_{1 n}\right) \\
\vdots & \vdots & \vdots \\
\Phi\left(\pi_{n 1}\right) & \cdots & \Phi\left(\pi_{n n}\right)
\end{array}\right) \quad\left(\Phi \in X^{*}\right) .
$$

The following result shows that there is bijective correspondence between representations of $A$ subordinate to $X$ and those of $X^{*}$. The proof is straightforward and omitted.

Lemma 2.2. Let $A$ be a Banach algebra and $X$ a faithful, introverted subspace of $A^{*}$. The map $\pi \longrightarrow \widetilde{\pi}$ is a bijection between the set of all (equivalence classes of) continuous representations of $A$ on $H$ subordinate to $X$ and the set of all (equivalent 
classes of) $w^{*}$-continuous representations of $X^{*}$ on $H$. Moreover, $\pi$ is irreducible if and only if $\widetilde{\pi}$ is irreducible.

Clearly all continuous representations of $A$ are subordinate to $A^{*}$. It is known that the coordinate functions of uniformly bounded weakly continuous representations of (semi)topological semigroups are weakly almost periodic (cf. [5, Example 4.2.2(c)]). In addition, coordinate functions of finite-dimensional $*$-representations of $L^{1}(G)$ are almost periodic functions (Dixmier [11, Théorème 16.2.1], BakerFilali [3, Lemma 1]). In the next lemma, we obtain the analogous results for representations of Banach algebra. We point out that the result for topological semigroups cited above does not apply to representations of Banach algebras since these representations are not uniformly bounded.

Lemma 2.3. Let $A$ be a Banach algebra.

(i) Every continuous representation $\pi: A \longrightarrow \mathscr{L}(H)$ with respect to the norm topology of $\mathscr{L}(H)$ is subordinate to $W A P(A)$.

(ii) Every continuous representation $\pi: A \longrightarrow M_{n}(\mathbf{C})$ is subordinate to $A P(A)$.

Proof. (i) A proof of this fact can be found in Young [38, p. 102]. We give a slightly different proof for completeness. For $\xi, \eta \in H$, we need to show that the linear operator

$$
A \longrightarrow A^{*}, \quad a \mapsto a \cdot \pi_{\xi, \eta}=\pi_{\pi(a) \xi, \eta}
$$

is weakly compact. Let $M>0$ be such that $\|\pi(a)\| \leq M$ if $\|a\| \leq 1$. Thus if $\left(a_{n}\right)$ is a sequence in the closed unit ball of $A$, then by the compactness of closed bounded sets in the weak operator topology of $\mathscr{L}(H)$, it follows that there exists a subsequence $\left(a_{n_{i}}\right)$ and an operator $T \in \mathscr{L}(H)$ such that $\pi\left(a_{n_{i}}\right) \rightarrow T$ in the weak operator topology. To complete the proof, it remains to show that $a_{n_{i}} \cdot \pi_{\xi, \eta} \rightarrow \pi_{T \xi, \eta}$ in the weak topology of $\sigma\left(A^{*}, A^{* *}\right)$. Indeed, if $\Phi \in A^{* *}$, then we have

$$
\begin{aligned}
\lim _{i}\left\langle\Phi, \pi_{\pi\left(a_{n_{i}}\right) \xi, \eta}\right\rangle & =\lim _{i}\left(\widetilde{\pi}(\Phi)\left(\pi\left(a_{n_{i}}\right) \xi\right) \mid \eta\right) \\
& =\lim _{i}\left(\pi\left(a_{n_{i}}\right) \xi \mid \widetilde{\pi}(\Phi)^{*} \eta\right) \\
& =\left(T \xi \mid \widetilde{\pi}(\Phi)^{*} \eta\right) \\
& =(\widetilde{\pi}(\Phi)(T \xi) \mid \eta) \\
& =\left\langle\Phi, \pi_{T \xi, \eta}\right\rangle .
\end{aligned}
$$

Since $\Phi \in A^{* *}$ was arbitrary, statement (i) follows.

Statement (ii) can be proved similarly using the fact that closed bounded sets in $M_{n}(\mathbf{C})$ are compact in the norm topology.

We remark that by a result of Johnson [25] (see also [6, Theorem III.25.7]) the continuity of $\pi$ in Lemma 2.3 is automatically satisfied for all irreducible representations of $A$.

In the following we write $\left(X^{*}\right)^{n}$ to denote the Cartesian product $X^{*} \times \cdots \times X^{*}$ equipped with the usual coordinatewise operations. We view elements in $\left(X^{*}\right)^{n}$ as column vectors. We denote the transpose of a matrix $M$ by ${ }^{t} M$. The products . and $\square$ are defined in the introduction.

Lemma 2.4. Let $A$ be a Banach algebra and $X$ a faithful, introverted subspace of $A^{*}$. Let $\pi: A \longrightarrow M_{n}(\mathbf{C})$ be a representation of $A$ subordinate to $X$. Then for every $\bar{\Psi} \in\left(X^{*}\right)^{n}$ the following are equivalent: 
(i) $a \cdot \bar{\Psi}={ }^{t} \pi(a) \bar{\Psi}$, for all $a \in A$.

(ii) $\Phi \square \bar{\Psi}={ }^{t} \widetilde{\pi}(\Phi) \bar{\Psi}$, for all $\Phi \in X^{*}$.

Proof. We will show that (i) implies (ii) (the converse statement is clear). Note that $\left(X^{*}\right)^{n}$ is equipped with the product of $w^{*}$-topologies. Let $\Phi \in X^{*}$ and $\left(a_{\alpha}\right)$ be a net in $A$ such that $a_{\alpha} \rightarrow \Phi$ in the $w^{*}$-topology. By writing $\bar{\Psi}={ }^{t}\left(\Psi_{1}, \ldots, \Psi_{n}\right)$, and using the $w^{*}$-continuity of the map $\Phi \rightarrow \Phi \square \Psi$, we have

$$
\Phi \square \bar{\Psi}={ }^{t}\left(\Phi \square \Psi_{1}, \ldots, \Phi \square \Psi_{n}\right)=w^{*}-\lim _{\alpha} a_{\alpha} \cdot{ }^{t}\left(\Psi_{1}, \ldots, \Psi_{n}\right)=w^{*}-\lim _{\alpha} a_{\alpha} \cdot \bar{\Psi} .
$$

Hence by (i),

$$
\Phi \square \bar{\Psi}=w^{*}-\lim _{\alpha}{ }^{t} \pi\left(a_{\alpha}\right) \bar{\Psi}={ }^{t} \widetilde{\pi}(\Phi) \bar{\Psi} .
$$

Definition 2.5. An element $\bar{\Psi} \in\left(X^{*}\right)^{n}$ is called $\pi$-invariant if it satisfies either (and hence both) of the conditions of the above lemma.

Remark 2.6. The importance of this concept in the study of finite-dimensional left ideals in Banach algebras associated with locally compact groups was first realized in Filali [13. In that paper the first author introduced the concept of $U$-invariance for a continuous representation $U$ of a locally compact group $G$. Moreover, for the Banach algebra $L^{1}(G)^{* *}$ an additional concept of topological $U$-invariance was introduced in [13. For the case of $L^{1}(G)^{* *}$, the topological $U$-invariance is closely related (though, not identical) to our present concept of $\pi$-invariance.

Theorem 2.7. Let $A$ be a Banach algebra and $X$ a faithful, introverted subspace of $A^{*}$. Let $\pi: A \longrightarrow M_{n}(\mathbf{C})$ be a continuous representation subordinate to $X$, and let $\bar{\Psi}={ }^{t}\left(\Psi_{1}, \ldots, \Psi_{n}\right)$ be a non-zero $\pi$-invariant element in $\left(X^{*}\right)^{n}$. Then the subspace $M$ spanned by the vectors $\Psi_{1}, \ldots, \Psi_{n}$ is a left ideal of $X^{*}$ and $\operatorname{dim} M \leq$ $n$. Furthermore, $\operatorname{dim} M=n$, and $M$ is a minimal left ideal if and only if $\pi$ is irreducible.

Proof. Let $\Phi \in X^{*}$ and $1 \leq i \leq n$. Then

$$
\begin{aligned}
\Phi \square \Psi_{i} & =\operatorname{pr}_{i}(\Phi \square \bar{\Psi}) \\
& =\operatorname{pr}_{i}\left({ }^{(} \widetilde{\pi}(\Phi) \bar{\Psi}\right) \\
& =\operatorname{pr}_{i}\left(\left(\begin{array}{ccc}
\Phi\left(\pi_{11}\right) & \cdots & \Phi\left(\pi_{n 1}\right) \\
\vdots & \vdots & \vdots \\
\Phi\left(\pi_{1 n}\right) & \cdots & \Phi\left(\pi_{n n}\right)
\end{array}\right)\left(\begin{array}{c}
\Psi_{1} \\
\vdots \\
\Psi_{n}
\end{array}\right)\right) \\
& =\sum_{k=1}^{n} \Phi\left(\pi_{k i}\right) \Psi_{k} .
\end{aligned}
$$

Thus $\Phi \square \Psi_{i} \in M$ for all $\Phi \in X^{*}$ and all $1 \leq i \leq n$. Since every element of $M$ is a linear combination of $\Psi_{1}, \ldots, \Psi_{n}$, it follows that $M$ is a left ideal in $X^{*}$. It is clear that $\operatorname{dim} M \leq n$.

Now suppose $\pi$ is irreducible. We show that $M$ is minimal by proving that every non-zero $\Gamma \in M$ generates $M$. Let $\Gamma=\sum_{i=1}^{n} x_{i} \Psi_{i}=\mathbf{x} \cdot \bar{\Psi}$, where $\mathbf{x}$ is a vector in $\mathbf{C}^{n}$ with coordinates $x_{1}, \ldots, x_{n}$ (the new use of the symbol $\cdot$ to denote the bilinear map $\mathbf{x} \cdot \bar{\Psi}$ will not result in confusion with its use to denote the module action of $A$ on $X$ and $X^{*}$ ). Let $\Phi \in M$ so that $\Phi=\sum_{i=1}^{n} y_{i} \Psi_{i}=\mathbf{y} \cdot \bar{\Psi}$. Since $\pi$ is irreducible, 
for a suitable $a \in A$ we have $\pi(a) \mathbf{x}=\mathbf{y}$. Therefore

$$
\begin{aligned}
\Phi=\mathbf{y} \cdot \bar{\Psi} & =(\pi(a) \mathbf{x}) \cdot \bar{\Psi} \\
& =\mathbf{x} \cdot\left({ }^{t} \pi(a) \bar{\Psi}\right) \\
& =\mathbf{x} \cdot(a \cdot \bar{\Psi}) \\
& =a \cdot(\mathbf{x} \cdot \bar{\Psi})=a \cdot \Gamma .
\end{aligned}
$$

Next, we show that the set $\left\{\Psi_{1}, \ldots, \Psi_{n}\right\}$ is linearly independent in $X^{*}$ and hence $\operatorname{dim} M=n$. If for some $\mathbf{x} \neq 0$ in $\mathbf{C}^{n}, \mathbf{x} \cdot \bar{\Psi}=\sum_{i=1}^{n} x_{i} \Psi_{i}=0$, then from irreducibility of $\pi$ it follows that there exist $a_{j} \in A$ such that $\pi\left(a_{j}\right) \mathbf{x}=\mathbf{e}_{j},(j=1, \ldots, n)$, and therefore

$$
\Psi_{j}=\left(\pi\left(a_{j}\right) \mathbf{x}\right) \cdot \bar{\Psi}=\mathbf{x} \cdot\left({ }^{t} \pi\left(a_{j}\right) \bar{\Psi}\right)=\mathbf{x} \cdot\left(a_{j} \cdot \bar{\Psi}\right)=a_{j} \cdot(\mathbf{x} \cdot \bar{\Psi})=0 .
$$

Thus $\Psi_{j}=0$ for all $j$, contradicting our assumption that $\bar{\Psi} \neq 0$. Thus $\left\{\Psi_{1}, \ldots, \Psi_{n}\right\}$ is linearly independent.

It remains to prove the converse statement; that is, if $M$ is a minimal left ideal and $\operatorname{dim} M=n$, then $\pi$ is irreducible. Equivalently, we show that every non-zero vector $\mathbf{x} \in \mathbf{C}^{n}$ is a cyclic vector for $\pi$. Let $\Gamma=\mathbf{x} \cdot \bar{\Psi}=\sum_{i=1}^{n} x_{i} \Psi_{i}$. Then $A \cdot \Gamma$ is a subspace of $M$ and hence is finite-dimensional and $w^{*}$-closed. Thus

$$
A \cdot \Gamma=\overline{A \cdot \Gamma}^{w^{*}}=X^{*} \square \Gamma=M,
$$

where the last identity follows from the assumption that $M$ is minimal and of course $X^{*} \square \Gamma \subset M$ is a left ideal. Furthermore, for every $a \in A$,

$$
a \cdot \Gamma=a \cdot(\mathbf{x} \cdot \bar{\Psi})=\mathbf{x} \cdot(a \cdot \bar{\Psi})=\mathbf{x} \cdot\left({ }^{t} \pi(a) \Psi\right)=(\pi(a) \mathbf{x}) \cdot \bar{\Psi} .
$$

If $\mathbf{y} \in \mathbf{C}^{n}$, then it follows from (1) that there exists $a \in A$ such that $a \cdot \Gamma=\mathbf{y} \cdot \bar{\Psi}$, and it follows from (2) that

$$
(\pi(a) \mathbf{x}) \cdot \bar{\Psi}=a \cdot \Gamma=\mathbf{y} \cdot \bar{\Psi} .
$$

Linear independence of $\left\{\Psi_{1}, \ldots, \Psi_{n}\right\}$ (which is a consequence of the assumption that $\operatorname{dim} M=n$ ) implies that $\pi(a) \mathbf{x}=\mathbf{y}$, proving that $\mathbf{x}$ is a cyclic vector for $\pi$.

The converse of the above theorem is also true.

Theorem 2.8. Let $A$ be a Banach algebra and $X$ a faithful, introverted subspace of $A^{*}$. Let $M$ be an $n$-dimensional left ideal in $X^{*}$. Then there exist a continuous representation $\pi: A \longrightarrow M_{n}(\mathbf{C})$ subordinate to $X$ and a $\pi$-invariant element $\bar{\Psi} \in$ $\left(X^{*}\right)^{n}, \bar{\Psi}={ }^{t}\left(\Psi_{1}, \ldots, \Psi_{n}\right)$, such that $M=\operatorname{lin}\left\{\Psi_{1}, \ldots, \Psi_{n}\right\}$.

Proof. Let $\left\{\Psi_{1}, \ldots, \Psi_{n}\right\}$ be a basis for $M$. Given $\Phi \in X^{*}$ and $1 \leq j \leq n$, we have $\Phi \square \Psi_{j} \in M$ and hence $\Phi \square \Psi_{j}=\sum_{i=1}^{n} \alpha_{i j}(\Phi) \Psi_{i}$, where $\alpha_{i j}(\Phi) \in \mathbf{C}$. It is clear that each $\alpha_{i j}$ defines a linear functional on $X^{*}$. Below we shall verify that $\alpha_{i j}$ is $w^{*}$-continuous and hence $\alpha_{i j} \in X$.

If $\Phi_{\alpha}$ is a net in $X^{*}$ converging in the $w^{*}$-topology to $\Phi \in X^{*}$, then for each $j$, $\Phi_{\alpha} \square \Psi_{j} \rightarrow \Phi \square \Psi_{j}$, and hence,

$$
\sum_{i=1}^{n} \alpha_{i j}\left(\Phi_{\alpha}-\Phi\right) \Psi_{i}=\left(\Phi_{\alpha}-\Phi\right) \square \Psi_{j} \rightarrow 0 .
$$


Since $\Psi_{1}, \ldots, \Psi_{n}$ are linearly independent, it follows that for given $1 \leq k \leq n, \Psi_{k}$ does not annihilate $\bigcap_{j \neq k} \operatorname{ker} \Psi_{j}$ (cf. [7, Proposition A.1.4]), and hence there exists $f_{k} \in \bigcap_{j \neq k} \operatorname{ker} \Psi_{j}$ such that $\Psi_{k}\left(f_{k}\right)=1$. Hence using (3) we can write

$$
\begin{aligned}
\lim _{\alpha} \alpha_{k j}\left(\Phi_{\alpha}-\Phi\right) & =\lim _{\alpha} \sum_{i=1}^{n} \alpha_{i j}\left(\Phi_{\alpha}-\Phi\right)\left\langle\Psi_{i}, f_{k}\right\rangle \\
& =\lim _{\alpha}\left\langle\left(\Phi_{\alpha}-\Phi\right) \square \Psi_{j}, f_{k}\right\rangle \\
& =0
\end{aligned}
$$

which proves $w^{*}$-continuity of $\alpha_{k j}$. Therefore $\alpha_{i j} \in X$ for all $1 \leq i, j \leq n$. Now it is easy to verify that

$$
\pi: A \longrightarrow M_{n}(\mathbf{C}), \quad \pi(a)=\left(\alpha_{i j}(a)\right)
$$

is a continuous representation subordinate to $X$ and that the vector $\bar{\Psi}=$ ${ }^{t}\left(\Psi_{1}, \ldots, \Psi_{n}\right)$ is indeed $\pi$-invariant.

Although Theorems 2.7 and 2.8 give complete characterization of finitedimensional left ideals in $X^{*}$, they do not guarantee the existence of such ideals, as the following example shows.

Example 2.9. Let $G$ be a discrete non-amenable group. By Filali 13, Corollary of Theorem 3], $l^{1}(G)^{* *}$ has no finite-dimensional left ideals other than possibly those that are generated by right annihilators. But $l^{1}(G)^{* *}$ has no right annihilator since it contains an identity given by the point mass measure at the identity of $G$. Consequently, $l^{1}(G)^{* *}$ has no non-trivial finite-dimensional left ideals.

Our objective in the remainder of this section is to show an interesting link between the cohomological property of character amenability and the existence of finite-dimensional left ideals in $X^{*}$. More precisely, we show that if $A$ is nonradical commutative character amenable, then non-trivial finite-dimensional left ideals exist in $X^{*}$, and they are all direct sums of 1-dimensional left ideals generated by $\phi$-invariant elements in $X^{*}$, where $\phi \in \sigma(A) \cup\{0\}$.

It follows immediately from the definition of character amenability that if $\phi, \psi \in$ $\sigma(A) \cup\{0\}$ and if $d: A \longrightarrow \mathbf{C}$ is a continuous linear map such that

$$
d(a b)=\phi(a) d(b)+d(a) \psi(b) \quad(a, b \in A),
$$

then $d=0$ (we call $d$ a continuous derivation into ${ }_{\phi} \mathbf{C}_{\psi}$ ). The following lemma is important for our purposes.

Lemma 2.10. Let $A$ be a commutative character amenable Banach algebra and $\pi: A \longrightarrow M_{n}(\mathbf{C})$ be a continuous representation. Then $\pi \simeq \pi^{\prime}$, where

$$
\pi^{\prime}(a)=\left(\begin{array}{cccc}
\phi_{1}(a) & 0 & \cdots & 0 \\
0 & \phi_{2}(a) & \cdots & 0 \\
\vdots & \vdots & \ddots & \vdots \\
0 & 0 & \cdots & \phi_{n}(a)
\end{array}\right) \quad(a \in A)
$$

and $\phi_{1}, \ldots, \phi_{n} \in \sigma(A) \cup\{0\}$. Moreover, if $X$ is a faithful introverted subspace of $A^{*}$, then $\pi$ is subordinate to $X$ if and only if $\pi^{\prime}$ is so. 
The first part of this result, under the assumptions that $A$ is a commutative Banach algebra and $(\operatorname{ker} \phi)^{2}$ is dense in $\operatorname{ker} \phi$ for all $\phi \in \sigma(A)$, may be found in Bade, Dales, and Lykova [2, p. 57]. It should be noted that our assumptions are in fact equivalent to the latter assumptions for the Banach algebras $A_{p}(G)$ and $L^{1}(G)$ and that they are only slightly stronger in general 37, Corollaries 2.4 and 2.7]. Below we shall give an alternative and simple proof of Lemma 2.10

Proof. Since $A$ is commutative the matrices $\pi(a), a \in A$, commute, and thus in a suitable basis of $\mathbf{C}^{n}$ we may assume that every $\pi(a)$ is upper-triangular [36]. Hence we may write

$$
\pi(a)=\left(\begin{array}{cccc}
\alpha_{11}(a) & \alpha_{12}(a) & \cdots & \alpha_{1 n}(a) \\
0 & \alpha_{22}(a) & \cdots & \alpha_{2 n}(a) \\
0 & 0 & \cdots & \alpha_{3 n}(a) \\
\vdots & \vdots & \ddots & \vdots \\
0 & 0 & \cdots & \alpha_{n n}(a)
\end{array}\right) \quad(a \in A)
$$

where $\alpha_{i j} \in A^{*}$ for all $i \leq j$ and $\alpha_{i i} \in \sigma(A) \cup\{0\}$ for $i=1, \ldots, n$. We use induction to show that for $i<j, \alpha_{i j}$ is a continuous derivation of the form (4) and therefore it must be zero. Let $1 \leq i<n$ be fixed. Using (6) and the identity $\pi(a b)=\pi(a) \pi(b)$ we have

$$
\alpha_{i i+1}(a b)=\alpha_{i i}(a) \alpha_{i+1}(b)+\alpha_{i i+1}(a) \alpha_{i+1 i+1}(b),
$$

from which it follows that $\alpha_{i i+1}=0$. Let us assume that $\alpha_{i i+k}=0$, for $k=$ $1,2, \ldots, l-1<n-i$. Then once again we may write

$$
\alpha_{i i+l}(a b)=\sum_{k=0}^{l} \alpha_{i i+k}(a) \alpha_{i+k i+l}(b)=\alpha_{i i}(a) \alpha_{i i+l}(b)+\alpha_{i i+l}(a) \alpha_{i+l i+l}(b),
$$

and hence $\alpha_{i i+l}=0$. This completes the proof of the first statement of the lemma. The proof of the second statement is straightforward and is omitted.

The following result follows from Lemma 2.10 and Theorem 2.8

Theorem 2.11. Let $A$ be a commutative character amenable Banach algebra and $X$ be a faithful introverted subspace of $A^{*}$. Then every finite-dimensional left ideal in $X^{*}$ is a direct sum of 1-dimensional left ideals generated by $\phi$-invariant elements where $\phi \in \sigma(A) \cup\{0\}$. If $X \cap \sigma(A) \neq \varnothing$, such ideals always exist in $X^{*}$.

Proof. Let $M$ be an $n$-dimensional left ideal in $X^{*}$. By Theorem 2.8 there exists a continuous representation $\pi: A \longrightarrow M_{n}(\mathbf{C})$ subordinate to $X$ and a $\pi$-invariant element $\bar{\Psi} \in\left(X^{*}\right)^{n}, \bar{\Psi}={ }^{t}\left(\Psi_{1}, \ldots, \Psi_{n}\right)$, such that $M=\operatorname{lin}\left\{\Psi_{1}, \ldots, \Psi_{n}\right\}$. By Lemma 2.10, $\pi \simeq \pi^{\prime}$, where $\pi^{\prime}$ is as in (5) and is subordinate to $X$. If $U$ is an invertible matrix such that $\pi^{\prime}(a)=U \pi(a) U^{-1}(a \in A)$, then the vector $\bar{\Psi}^{\prime}=$ ${ }^{t}\left(U^{-1}\right) \bar{\Psi}$ is $\pi^{\prime}$-invariant. By writing $\bar{\Psi}^{\prime}={ }^{t}\left(\Psi_{1}^{\prime}, \ldots, \Psi_{n}^{\prime}\right)$, it follows easily that $\Psi_{i}^{\prime}$ is $\phi_{i}$-invariant $(i=1, \ldots, n)$ and that

$$
M=\operatorname{lin}\left\{\Psi_{1}, \ldots, \Psi_{n}\right\}=\operatorname{lin}\left\{\Psi_{1}^{\prime}, \ldots, \Psi_{n}^{\prime}\right\} .
$$

By discarding those $\Psi_{i}^{\prime}$ that are linearly dependent on the rest, we may assume without loss of generality that $\left\{\Psi_{1}^{\prime}, \ldots, \Psi_{n}^{\prime}\right\}$ is linearly independent and hence $M=$ $\bigoplus_{i=1}^{n} \mathbf{C} \Psi_{i}^{\prime}$, as we wanted to show.

If $\phi \in X \cap \sigma(A)$, then it follows from character amenability of $A$ that there exists a $\phi$-invariant element $\Phi \in A^{* *}$ such that $\Phi(\phi) \neq 0$ (37]). Then of course the 
canonical image of $\Phi$ in $X^{*}$ is non-zero and generates a 1-dimensional left ideal, proving the existence of such ideals in $X^{*}$.

Examples of commutative character amenable Banach algebras $A$ for which Theorem 2.11 is applicable include (a) commutative $\mathrm{C}^{*}$-algebras; (b) the group algebra $L^{1}(G)$ for $G$ commutative; more generally (c) the Herz-Figà-Talamanca algebras $A_{p}(G)$ for $G$ amenable; (d) any uniform algebra $A(X)$ where $X$ coincides with the Choquet boundary of $A$ ([37, 24]). In view of Theorem [1.1, the situation for $A(G)$ (or more generally for $A_{p}(G)$ ) is of particular interest, which we state in the following corollary.

Corollary 2.12. If $G$ is an amenable locally compact group, then all finitedimensional left ideals in $A_{p}(G)^{* *}$ and in $U C_{p}(G)^{*}$ are generated by $\phi$-invariant elements.

Remark 2.13. If $\phi=0$ is the zero character, an element $\Phi_{0} \in A^{* *}$ is $\phi$-invariant if and only if it is a right annihilator in $A^{* *}$. The existence of such elements is

equivalent to the condition that $\varlimsup_{\ln \left(A^{*} \cdot A\right)}^{\|\cdot\|}$ is a proper subspace of $A^{*}$. This is the case, for example, if $G$ is a non-discrete locally compact group and $A=L^{1}(G)$. In this case, $L^{\infty}(G) \cdot L^{1}(G)=C_{r u}(G) \neq L^{\infty}(G)$ (cf. [22, (32.45)(b)]), and hence $L^{1}(G)^{* *}$ has finite-dimensional left ideals generated by right annihilators. It is shown in Filali [13, Corollary in p. 2332] that finite-dimensional left ideals which are not generated by right annihilators exist in $L^{1}(G)^{* *}$ if and only if $G$ is amenable. This however is not true in $A_{p}(G)^{* *}$ and $U C_{p}(G)^{*}$ since invariant means always to exist in these algebras, and so 1-dimensional left ideals exist without $G$ being amenable. It is interesting to compare these results with Theorem 3.5 of LauWong 34, in which it is shown that $L^{1}(G)$ satisfies a finite-dimensional invariant subspace property called $T(n)$, for $n=1,2, \ldots$, if and only if $G$ is amenable, while the same property is always satisfied by the Fourier algebra $A(G)$, for the same reason that was alluded to above. We thank the referee for bringing reference [34] to our attention.

\section{Finite-Dimensional ideAls IN REGULAR BANACH FUNCTION ALGEBRAS}

In this section we show that for a large class of Banach algebras of interest in harmonic analysis, that is, for commutative semisimple Banach algebras, there exists a complete characterization of finite-dimensional ideals in terms of minimal idempotents.

Recall that a minimal idempotent in an algebra $A$ is a non-zero element $w$ such that $w^{2}=w$ and $w A w=\mathbf{C} w$. It is known that for semiprime algebras (that is, algebras for which $\{0\}$ is the only two sided ideal $J$ such that $J^{2}=\{0\}$ ), minimal idempotents determine all minimal left (or right) ideals. More precisely, $M$ is a minimal left (right) ideal in $A$ if and only if $M=A w(M=w A)$ for some minimal idempotent $w \in A$ (cf. [6, Proposition IV.30.6]). We denote the Gelfand transform of $w \in A$ by $\widehat{w}$.

Theorem 3.1. Let $A$ be a commutative Banach algebra. If $w \in A$ is a minimal idempotent, then there exists $\psi \in \sigma(A)$ such that $\widehat{w}=\chi_{\{\psi\}}$ is the characteristic function of the singleton $\{\psi\}$. The converse is true if $A$ is semisimple. 
Proof. Let $w \in A$ be a minimal idempotent. Since $A$ is commutative, it follows that $A w=\mathbf{C} w$. Therefore for each $v \in A, v w=\psi(v) w$, where $\psi: A \longrightarrow \mathbf{C}$ is a non-zero homomorphism and $\psi(w)=1$. Hence $\psi \in \sigma(A)$ and $\widehat{w}(\psi)=1$. Next we check that $\widehat{w}(\phi)=0$ for all $\phi \neq \psi$. Let $v \in A$ be such that $\phi(v) \neq \psi(v)$. Then

$$
\phi(v) \phi(w)=\phi(v w)=\phi(\psi(v) w)=\psi(v) \phi(w),
$$

which implies that $\phi(w)=0$. Hence $\widehat{w}=\chi_{\{\psi\}}$.

The converse statement is clear since under our assumptions $A \cong \widehat{A}$, and of course $\widehat{w}$ is a minimal idempotent in $\widehat{A}$.

Corollary 3.2. If $A$ is a commutative semisimple Banach algebra, then all finitedimensional ideals in $A$ are generated by minimal idempotents.

Proof. For simplicity of notation we may identify $A$ with its image under the Gelfand transform, and by transferring the norm, we may assume that $A$ is a Banach function algebra on a locally compact space $X$ and $\sigma(A)=\left\{\tau_{x}: x \in X\right\}$, where $\tau_{x}$ is the evaluation functional at $x$. Now suppose that $I$ is a finite-dimensional ideal in $A$. Then either $I$ is minimal or it contains a minimal ideal. Since $A$ is semiprime, it follows that $I$ contains a minimal idempotent $\chi_{\{x\}}$ with $x \in X$ (Theorem 3.1). Let $\left\{\chi_{\left\{x_{1}\right\}}, \ldots, \chi_{\left\{x_{n}\right\}}\right\}$ be a maximal set of minimal idempotents in $I$ (the set is finite since its members are linearly independent). Let $J=\operatorname{lin}\left\{\chi_{\left\{x_{1}\right\}}, \ldots, \chi_{\left\{x_{n}\right\}}\right\}$. If $J=I$ we have nothing to prove; otherwise let $v \in I-J$ and define $J^{\prime}=$ $\left\{v \in I: v\left(x_{i}\right)=0, i=1, \ldots, n\right\}$. Note that $v-\sum_{i=1}^{n} v\left(x_{i}\right) \chi_{\left\{x_{i}\right\}} \in J^{\prime}$, and hence $J^{\prime} \neq\{0\}$. Since $J^{\prime}$ is a finite-dimensional ideal in $A$, it follows that it must contain a minimal idempotent $\chi_{\left\{x_{0}\right\}} \in I$, contradicting the maximality of $\left\{\chi_{\left\{x_{1}\right\}}, \ldots, \chi_{\left\{x_{n}\right\}}\right\}$. The contradiction proves that $I=J$.

Corollary 3.3. Let $G$ be a locally compact group and $A$ be a Banach function algebra on $G$ whose minimal idempotents (if they exist) are characteristic functions $\chi_{\{x\}}(x \in G)$. If $A$ has any non-trivial finite-dimensional ideal, then $G$ is discrete. Conversely, if $A$ is regular and $G$ is discrete, then finite-dimensional left ideals exist and they are exactly of the form $\operatorname{lin}\left\{\chi_{\left\{x_{1}\right\}}, \ldots, \chi_{\left\{x_{1}\right\}}\right\}$.

Proof. If $A$ has a finite-dimensional ideal, then by Corollary 3.2 , $A$ must have a minimal idempotent $\chi_{\{x\}}$ for some $x \in G$. This implies that $\{x\}$ is an open set and hence $G$ is discrete.

On the other hand, assuming $G$ is discrete and $A$ is regular, for every closed subset $S \subset G$ and every $x \notin S$ there exists a function $f \in A$ such that $f(x)=1$ and $f(S)=\{0\}$. In particular, $\chi_{\{x\}} \in A$ for all $x \in G$. These are all the minimal idempotents by Theorem 3.1. So by Corollary 3.2. finite-dimensional ideals are exactly of the form $\operatorname{lin}\left\{\chi_{\left\{x_{1}\right\}}, \ldots, \chi_{\left\{x_{1}\right\}}\right\}$, where $x_{1}, \ldots, x_{n} \in G$.

Examples 3.4. Examples of Banach function algebras on $G$ whose minimal idempotents (if they exist) are characteristic functions $\chi_{\{x\}}(x \in G)$ include all natural Banach function algebras on $G$. Recall that a Banach function algebra $A$ on $G$ is natural if $\sigma(A)=\left\{\tau_{x}: x \in G\right\}$, where $\tau_{x}$ is the evaluation functional at $x$ ([8, Chapter 4]). It is well known that $C_{0}(G)$ and $A_{p}(G)(1<p<\infty)$ are natural. Non-natural Banach function algebras satisfying the same condition include $W_{p}(G), B_{p}(G)$, or $M A_{p}(G)$ (see Derighetti et al. [10, Theorem 5.5]). We refer to the last reference as well as Granirer [20] for definitions and other properties of these spaces. 


\section{ACKNOWLEDGEMENTS}

The authors would like to thank M. Neufang for stimulating discussions and the referee for a careful reading of this paper and for helpful suggestions.

\section{REFERENCES}

[1] R. Arens, The adjoint of a bilinear operation, Proc. Amer. Math. Soc. 2 (1951), 839-848. MR0045941 (13:659f)

[2] W. G. Bade, H. G. Dales, and Z. A. Lykova, Algebraic and strong splittings of extensions of Banach algebras, Mem. Amer. Math. Soc. 656 (1999). MR1491607 (99g:46059)

[3] J. W. Baker and M. Filali, Minimal ideals in group algebras and their biduals, Math. Proc. Cambridge. Phil. Soc. 120 (1996), 475-488. MR.1388201 (98f:22004)

[4] - On minimal ideals in some Banach algebras associated with locally compact groups, J. London Math. Soc. 63 (2001), 83-98. MR1802759 (2002m:43003)

[5] J. F. Berglund, H. D. Junghenn, and P. Milnes, Analysis on Semigroups, John Wiley \& Sons, New York, 1989. MR.999922 (91b:43001)

[6] F. F. Bonsall and J. Duncan, Complete Normed Algebras, Springer, New York, 1973. MR0423029 (54:11013)

[7] J. B. Conway, A Course in Functional Analysis, Second edition, Springer, New York, 1990. MR1070713 (91e:46001)

[8] H. G. Dales, Banach Algebras and Automatic Continuity, Oxford University Press, Oxford, 2000. MR1816726 (2002e:46001)

[9] H. G. Dales and A. T.-M. Lau, The second duals of Beurling algebras, Mem. Amer. Math. Soc. 836 (2005). MR2155972 (2006k:43002)

[10] A. Derighetti, M. Filali, and M. Sangani Monfared, On the ideal structure of some Banach algebras related to convolution operators on $L^{p}(G)$, J. Funct. Analysis 215 (2004), 341-365. MR2151297 (2008b:43014)

[11] J. Dixmier, Les $C^{*}$-Algèbres et Leurs Représentations, Deuxième èdition, Éditions Jacques Gabay, Paris, 1996. MR1452364 (98a:46066)

[12] P. Eymard, L'algèbre de Fourier d'un groupe localement compact, Bull. Soc. Math. France 92 (1964), 181-236. MR0228628 (37:4208)

[13] M. Filali, Finite-dimensional left ideals in some algebras associated with a locally compact group, Proc. Amer. Math. Soc. 127 (1999), 2325-2333. MR1487366 (99j:22005)

[14] M. Filali, M. Neufang, and M. Sangani Monfared, On ideals in the bidual of the Fourier algebra and related algebras, J. Funct. Analysis 258 (2010), 3117-3133. MR2595737

[15] _ Weak factorizations of operators in the group von Neumann algebras of certain amenable groups and applications, Math. Annalen (to appear).

[16] M. Filali and J. S. Pym, Right cancellation in LUC-compactification of a locally compact group, Bull. London. Math. Soc. 35 (2003), 128-134. MR1935082 (2003i:22006)

[17] M. Filali and P. Salmi, One-sided ideals and right cancellation in the second dual of the group algebra and similar algebras, J. London Math. Soc. 75 (2007), 35-46. MR2302728 (2009f:43003)

[18] Slowly oscillating functions in semigroup compactifications and convolution algebras, J. Funct. Analysis. 250 (2007), 144-166. MR2345910 (2009b:43001)

[19] F. Ghahramani and A. T.-M. Lau, Multipliers and ideals in second conjugate algebras related to locally compact groups, J. Funct. Analysis 132 (1995), 170-191. MR.1346222 (97a:22005)

[20] E. E. Granirer, On some spaces of linear functionals on the algebra $A_{p}(G)$ for locally compact groups, Colloq. Math. LII (1987), 119-132. MR891504 (88k:43006)

[21] C. Herz, Harmonic synthesis for subgroups, Ann. Inst. Fourier (Grenoble) 23 (1973), 91-123. MR0355482 (50:7956)

[22] E. Hewitt and K. A. Ross, Abstract Harmonic Analysis, Vol. 2, Springer-Verlag, New York, 1970. MR0262773(41:7378)

[23] Z. Hu, On the set of topologically invariant means on the von Neumann algebra $V N(G)$, Illinois J. Math. 39 (1995), 463-490. MR.1339838 (96e:22012)

[24] Z. Hu, M. Sangani Monfared, and T. Traynor, On character amenable Banach algebras, Studia Math. 193 (2009), 53-78. MR 2506414(2010d:46060) 
[25] B. E. Johnson, The uniqueness of the (complete) norm topology, Bull. Amer. Math. Soc. 73 (1967), 537-539. MR0211260 (35:2142)

[26] E. Kaniuth, A. T.-M. Lau, and J. Pym, On $\phi$-amenability of Banach algebras, Math. Proc. Cambridge Phil. Soc. 144 (2008), 85-96. MR2388235 (2009a:46084)

[27] A. T.-M. Lau, Uniformly continuous functionals on the Fourier algebra of any locally compact group, Trans. Amer. Math. Soc. 251 (1979), 39-59. MR531968 (80m:43009)

[28] - The second conjugate algebra of the Fourier algebra of a locally compact group, Trans. Amer. Math. Soc. 267 (1981), 53-63. MR621972 (83e:43009)

[29] - Continuity of Arens multiplication on the dual space of bounded uniformly continuous functions on locally compact groups and topological semigroups, Math. Proc. Cambridge Phil. Soc. 99 (1986), 273-283. MR817669(87i:43001)

[30] Uniformly continuous functionals on Banach algebras, Colloquium Math. 51 (1987), 195-205. MR891287 (88f:43006)

[31] A. T.-M. Lau and V. Losert, On the second conjugate algebra of $L^{1}(G)$ of a locally compact group, J. London Math. Soc. 37 (1988), 464-470. MR.939122 (89e:43007)

[32] _ The $C^{*}$-algebra generated by the operators with compact support on a locally compact group, J. Funct. Analysis. 112 (1993), 1-30. MR.1207935 (94d:22005)

[33] - The centre of the second conjugate algebra of the Fourier algebra for infinite products of groups, Math. Proc. Cambridge Phil. Soc. 138 (2005), 27-39. MR2127225 (2006c:43003)

[34] A. T.-M. Lau and J. C. S. Wong, Invariant subspaces for algebras of linear operators and amenable locally compact groups, Proc. Amer. Math. Soc. 102 (1988), 581-586. MR928984 (89c:43007)

[35] M. Neufang, A unified approach to the topological centre problem for certain Banach algebras arising in abstract harmonic analysis, Arch. Math. 82 (2004), 164-171. MR2047670 (2005g:22004)

[36] H. Radjavi and P. Rosenthal, Simultaneous Triangulation, Springer-Verlag, New York, 2000. MR:1736065 (2001e:47001)

[37] M. Sangani Monfared, Character amenability of Banach algebras, Math. Proc. Cambridge Phil. Soc. 144 (2008), 697-706. MR2418712 (2009b:46104)

[38] N. J. Young, Periodicity of functionals and representations of normed algebras on reflexive spaces, Proc. Edinburgh Math. Soc. 20 (1976), 99-120. MR0435849 (55:8800)

Department of Mathematical Sciences, University of Oulu, Oulu 90014, Finland

E-mail address: mahmoud.filali@oulu.fi

Department of Mathematics and Statistics, University of Windsor, Windsor, ON, N9B 3P4, CANADA

E-mail address: monfared@uwindsor.ca 